\title{
Addressing Healthcare Needs in Older People after Discharge
}

\author{
Maria Grazia Proietti ${ }^{1}$, Teresa Compagnone ${ }^{2}$, Giuseppe Liotta $^{3^{*}}$ \\ ${ }^{1}$ Community Cooperative Nuova Sair, Rome, Italy \\ ${ }^{2}$ University of Tor Vergata, Rome, Italy \\ ${ }^{3}$ Biomedicine and Prevention Department, University of Tor Vergata, Rome, Italy \\ Email: ${ }^{*}$ giuseppe.liotta@uniroma2.it
}

Received 10 May 2014; revised 22 June 2014; accepted 27 July 2014

Copyright (C) 2014 by authors and Scientific Research Publishing Inc.

This work is licensed under the Creative Commons Attribution International License (CC BY). http://creativecommons.org/licenses/by/4.0/

(c) () D Den Access

\begin{abstract}
Healthcare needs should be addressed even when patients return home. The purpose of this study was to support a multidimensional assessment upon discharge to plan domiciliary care. Materials and Methods: This is a multicentre cross-sectional study that uses the Geriatric Functional Assessment questionnaire to assess healthcare needs. The questionnaire was administered upon discharge to 358 people aged over 65 . Results: About $20 \%$ of our sample required healthcare support after discharge and $32 \%$ needed some sort of supervision. Most of the healthcare requirements involved socio-economic needs, neurological diseases, and inability to autonomously perform instrumental activities of daily living. Discussion: It is crucial to have an optimal discharge plan for older inpatients. Nurses play a key role in managing the care continuum process, and they have the instruments to select those requiring "supported discharge", and collaborate with district nurses who can arrange healthcare interventions jointly with other professionals. They can facilitate access to resources, medical devices and aids required to restore patient autonomy.
\end{abstract}

\section{Keywords}

Multidimensional Evaluation, Elderly, Hospital Discharge

\section{Introduction}

Ageing of the population has become a priority for healthcare services worldwide, especially in industrialised countries, and Italy in particular is one the countries with the highest ageing rate, where more than $20 \%$ of its entire population is aged over 65 [1]. The healthcare relevance of ageing lies in the fact that, in addition to the physiological ageing of individuals, an increased incidence of co-morbidity relates to older people's demand of care.

${ }^{*}$ Corresnonding author 
A particularly vulnerable time for older people discharged from hospital when nurses have to decide whether home care needs should be provided. Nevertheless, healthcare continuum called upon by the Project for Older People's Good Health [2], today still continues to be very challenging. According to this project, healthcare needs in older patients are determined according to the methodology of multidimensional assessment. Nevertheless, upon discharge this is totally missing and the discharge letter is often the only document to describe the patients' healthcare needs, independently from the patients' ability to satisfy those needs.

\section{Background}

Between the end of the 1960s and the beginning of the 1980s Rubenstein [3] sensed the advantage of adapting the use of healthcare resources (within hospitals and in the community) to the conditions and the healthcare needs of each patient. In this way, it was possible to optimize the cost/benefit ratio and at the same time improve outcomes, both in terms of health and quality of life. Rubenstein tested the relevance of this intuition by comparing two groups of patients discharged from hospital: the first group followed the usual community care procedure, whereas the second group was cared for according to the indications that emerged from the Multidimensional Functional Assessment (MFA), a tool that measures patient autonomy and need for care, taking into account not just the disease they suffer from, but also their psycho-functional abilities and their social and financial resources. During the period of observation, the functional abilities in the experimental group improved, and the number and duration of admissions diminished, as well as the mortality rates. Initially in the United States and then also in Europe, MFA became the most commonly used tool to asses health conditions and healthcare needs in older patients.

In fact, discharge from hospital is often a problematic experience for older patients, because the illness that led to admission and the hospitalization itself in many cases entails the so-called "functional decline". Aggravated by the pressure to reduce the length of stay in hospitals, older people are increasingly discharged in conditions of incomplete recovery. Deschodt et al. [4] defined functional decline as "increase of the Katz Index ${ }^{1} 30$ days after discharge", even by just one unit compared to the score obtained before admission. The researchers observed this condition in 279 out of 752 patients aged over 75 years discharged from hospital (39\%). The first step to prevent functional decline is to identify patients at risk, followed by the implementation of targeted interventions. Thanks to their patient observation and healthcare planning skills, nurses can play a key role in this process.

Hoogerduijn et al. [5], starting from the modest effectiveness shown by the screening tools designed to identify hospitalized patients at risk of post-discharge functional decline (Hospital Admission Risk Profile, Identification of Seniors at Risk, Care Complexity Prediction Instrument), identified some specific predictors, such as age, low functional level, cognitive deterioration, pre-admission inability to perform Instrumental Activities of Daily Living (IADLs), depression and the length of stay in hospital.

Also Ferioli et al. [6] investigated the factors that mostly entailed conditions of dependence in older patients. The variables they took into account were: age, functional ability, cognitive deterioration, nutritional status $(\mathrm{BMI})^{2}$, and the number of concomitant diseases. They found that poor functional abilities and serious cognitive deterioration were the main factors that determined a high level of dependency in older people $(\mathrm{p}<0.001)$. From their univariate correlation analysis they found that age influenced healthcare dependence, as well as BMI, whereas multivariate analysis showed how only cognitive deterioration, functional status, and BMI maintained a high statistical significance. The authors concluded that it is not ageing in itself that determines healthcare dependence in older patients, but age is significant only if correlated with cognitive, functional, and nutritional status.

On the basis of a review of retrospective, prospective, and mixed studies focusing on planning discharge for older hospitalized patients, Bowles et al. [7] came to the conclusion that older people with intermittent or no domiciliary support, with difficulty walking, poor awareness of their own health conditions, long hospital stay, depression, and a large number of comorbidities should have the priority to be receive post-discharge services.

In a more recent study, Hoogerduijn et al. [8] developed the Identification of Seniors at Risk-Hospitalized Pa-

\footnotetext{
${ }^{1}$ A scale that measures Independence of Activities of Daily Living proposed by Katz et al. in 1963. It assesses 6 basic activities: bathing, dressing, toileting, transferring, continence, and feeding.

${ }^{2}$ Body Mass Index = peso $(\mathrm{Kg}) /$ height $(\mathrm{m})^{2}$. BMI $<19$ (underweight), BMI = $19-24$ (average), BMI = $25-30$ (overweight), BMI $>30$ (obese).
} 
tients (ISAR-HP), a predictive model with only 4 items: the need for support for IADL, support for walking, support to go outdoors, and leaving school before the age of 14. ISAR-HP is a screening tool that is easy and quick to use to identify older people at risk of functional decline after discharge from hospital.

\subsection{Aim}

The purpose of our study is to measure the effectiveness of a multidimensional tool in assessing healthcare needs upon discharge of older patients in order to plan their care at home.

\subsection{Materials and Methods}

During the practical session of the Blended Online Course on Methodology of Nursing Research, organized by the IPASVI Nursing Board in Rome as one of the projects of the Centre of Excellence for Nursing Scholarship, learners were asked to administer the questionnaire for Geriatric Functional Assessment to a population aged over 65 discharged from hospital to identify their healthcare needs. The questionnaire was developed from the Grauer Functional Rating Scale [9], validated in Italian by Palombi et al. [10] [11].

The scale consists of seven sections, plus an introductory one regarding the socio-demographic data. The seven sections assess: physical conditions, mental conditions, functional ability (ADL/IADL), community support, type of home, social relations, and financial situation. For each section, a synthetic score and a total score were calculated, which could range from -108 to +101 , informing three types of decisions on healthcare needs:

$$
<10 \text { : the patient urgently needs healthcare services at home or residence; }
$$

10 - 50: the patient does not urgently need healthcare services, but requires active monitoring due to specific vulnerabilities;

>50: the patient does not need healthcare support or monitoring, apart from periodic assessment of autonomy.

Interviewers were taught how to use the tool by the researchers of the Laboratory of Epidemiology of the University of Rome Tor Vergata. Data were collected between December 2011 and March 2012.

It took 15 - 20 minutes to complete each questionnaire. In case the patient was unable to answer, it was administered to their significant other.

\subsection{The Setting}

The interviewers were 29 nurses who attended the methodology course ${ }^{3}$, who administered the questionnaire to the patients admitted to the ward where they regularly worked, after obtaining the authorization from the hospital and consent from patients. The sample included 358 patients aged over 65 (Table 1), selected according to the following inclusion criteria:

- Patients being discharged from medical wards, not terminally-ill.

- Males and females aged $>65$ years.

The mean age of our sample was 78.2 years for women ( $\mathrm{SD} \pm 7.9$ ) and 76.6 years for men (SD \pm 7.2 ), evenly distributed between males and females (Table 2).

A statistical analysis was conducted to identify significant relations with the final score of the questionnaire. For this reason, all the variables of the questionnaire were compared with the final score recoded according to the three levels of healthcare needs described above. The variables that showed a statistically significant relation with the final score were included in a Multiple Correspondence Analysis, which consists of a factor analysis methodology aimed at studying mutual relationships between different variables within the sample, projecting its values on a two-dimensional plane. The distances between the variables represent the interpretative keys of the graph, which offer a visual representation of this analysis. Data analysis was performed with SPSS 19.0.

\footnotetext{
${ }^{3}$ Surveyors: Angelone Loredana, Brunelli Maurizio, Cannovo Maria, Carnevale Marinella, Cicala Carmelo, Colasanti Daniela, Diodato Anna Rita, Forte Daniela, Fraticelli Luciana, Giorgi Fabio, Graziano Lorenza, Guardiani Nadia, Iaquinta Francesco, Inforti Antonella, Landi Lidia, Martelli Sara, Napoli Cinzia, Nobis Beata, Onofri Claudia, Patarini Barbara, Pazzaglini Anna Rita, Pettenuzzo Eleonora, Popa Nicoleta, Punziano Antonella, Rubino Maria Michelina, Sacco Antonio Fabio, Scarioli Teresita, Soldà Laura, Venditti Giovanna.
} 
Table 1. The survey setting.

\begin{tabular}{ccc}
\hline Town & $\mathbf{N}^{\circ}$ of Questionnaires & \\
\hline Albano & 20 & Lazio (90) \\
Civitavecchia & 40 & \\
Genzano & 20 & Town In Southern Italy (121) \\
Subiaco & 10 & \\
Isernia & 17 & Rome (147) \\
Napoli & 14 & \\
Cosenza & 90 & \\
Roma & 358 & \\
TOTAL & 347 & \\
\hline
\end{tabular}

Table 2. Gender distribution and average age (gender) (average age) (SD).

\begin{tabular}{cccc}
\hline Gender & Average Age & N & SD \\
\hline Female & 78.2 & 178 & \pm 7.9 \\
Male & 76.6 & 179 & \pm 7.2 \\
Total & 77.4 & 357 & \pm 7.6 \\
\hline
\end{tabular}

\section{Results}

The characteristics of the family members who took care of their older person discharged from hospital offered particularly significant data from a healthcare perspective. In our sample, almost half of the patients lived with their spouse (46\%), 22\% with their children; about 20\% alone, $7 \%$ with a paid caregiver, and $4 \%$ had other cohabitation solutions (Graph 1).

Regarding the final score of the questionnaire, about $20 \%$ of the interviewees after discharge required further care and 32\% needed some sort of monitoring (Graph 2). These percentages were twice than those usually reported in a sample of non-hospitalized older people [12]. Our results depended on the time of the survey that is at the end of a period of stay in hospital, and were consistent with the results obtained by Deschodt et al. [4]. However, it is possible that temporary conditions, which require healthcare support for short periods, influence healthcare demand until older patients achieve a full psychophysical recovery after the episode that led to being admitted to hospital. For instance, even a short period in bed, no longer than a week, can sometimes be enough to allow patients to stand up again on their own, as they used to do before their admission. These are situations that can often be resolved in a short amount of time, even with only a month of domiciliary rehabilitation. In this sense, services provided after discharge even for a limited amount of time, such as supported discharge, can be an appropriate response to the healthcare needs expressed, but which in most cases is not provided.

If we consider the final score per geographic zone (Graph 3), we notice that in provinces and southern towns there is less demand for care compared to Rome ( $p=0.085)$, maybe due to a stronger social network that ensures better care for older people after discharge.

The distribution of the final score based on the healthcare profiles (Graph 4) offered us the possibility the make further considerations. For instance, it is evident that those who lived alone and required healthcare services, could not be discharged without a home care plan $(\mathrm{p}=0.001)$. Less than $4 \%$ of our sample benefited from an in-depth evaluation and a customized post-discharge healthcare plan to maximise the patient's full psychophysical recovery. Also patients requiring monitoring ought to be discharged only after deciding who is 


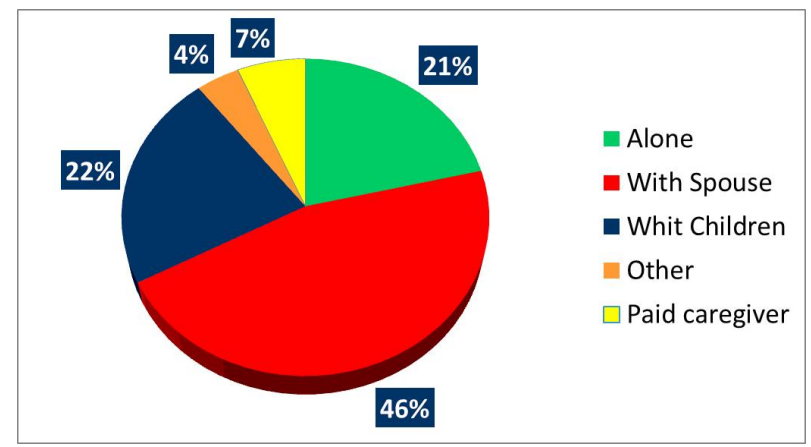

Graph 1. Distribution of cohabitation profiles.

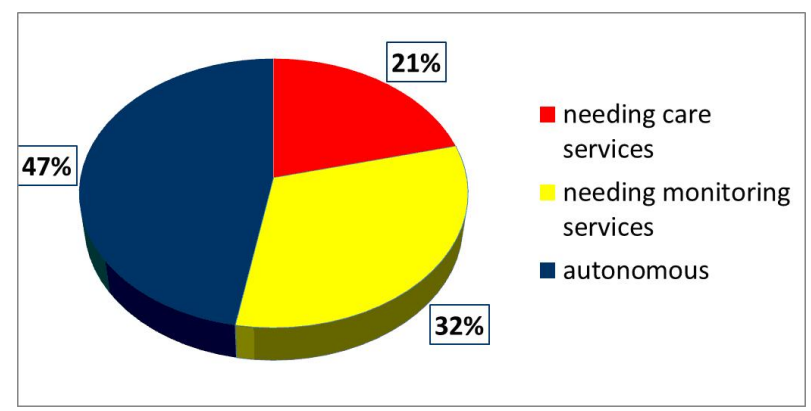

Graph 2. Distribution of final scores.

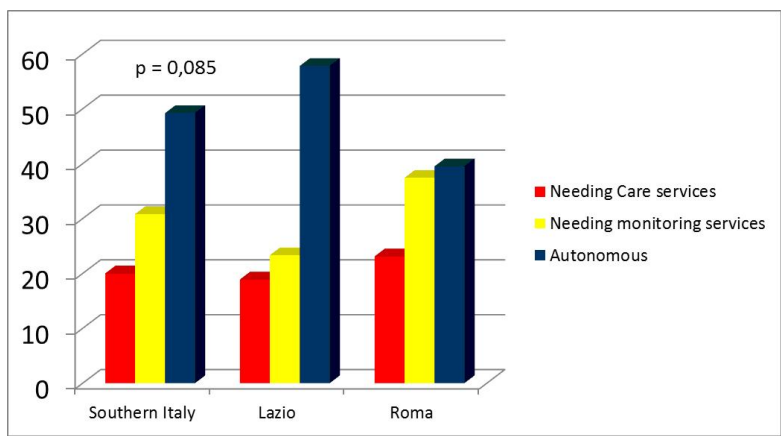

Graph 3. Final scores across geographical areas.

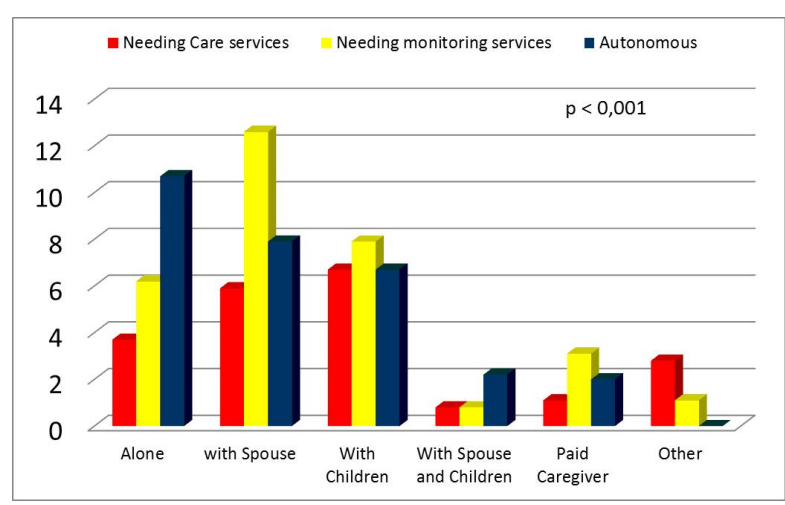

Graph 4. Final scores based on cohabitation profile.

going to monitor them, which may imply a phone call a day, or even less to check how they are. Particular attention during discharge needs to be paid also those who live only with their spouse, to see if they require post- 
discharge care or monitoring, because very often also their spouses are old and not fully autonomous. Finally, for all those requiring post-discharge care it was necessary to check whether the other family members could offer their support, to design a healthcare plan based on their needs and avoid that their clinical conditions deteriorated.

The application of the multiple correspondence analysis to the data of our study (Graph 5) allowed us to collected further information about the profile of older people needing care. In the graph, there is a line going from left to right, joining the two points, from the lowest score ( $<10$ top left-hand side) to the highest score ( $>50$ on the right), in the blue circles, whereas the score 10 - 50 is shown in between, towards the lower part of the graph. Two variables were strongly associated with this line: the cardio-respiratory function and mobility, with an inverted pattern: higher levels of mobility (walking outdoors) and cardiovascular functions are attributed a lower score (therefore to a condition requiring more care) and vice versa. This is probably an association conditioned by serious impairments in other areas, such the socioeconomic area or the association with an unclear cohabitation profile (defined as "other"). In fact, it is very unlikely that seriously impaired individuals manage to live in their homes if not fully supported by the other members of the family. The cohabitation profiles having a higher final score (meaning less need for care) are "living with your spouse" and "living with your spouse and children", associated with a solid financial situation.

Then there is another clearly-defined group of patients (red circle) that included: serious functional impairment, more than one neurological disorder, and the absence of psychic disorders. The scores inside the red circle express the worst conditions of the variables taken into account, but the patients are not disoriented or affected by loss of memory (the two "1s" in the circle). In addition, this group of patients lived with their children or a paid caregiver, and probably it was precisely the social-economical resources that made the difference. But this group, that showed the highest loss of physical autonomy, is positioned half way between the worst final score and the intermediate one, and therefore expresses a significant need for care, and therefore deserving particular attention upon discharge.

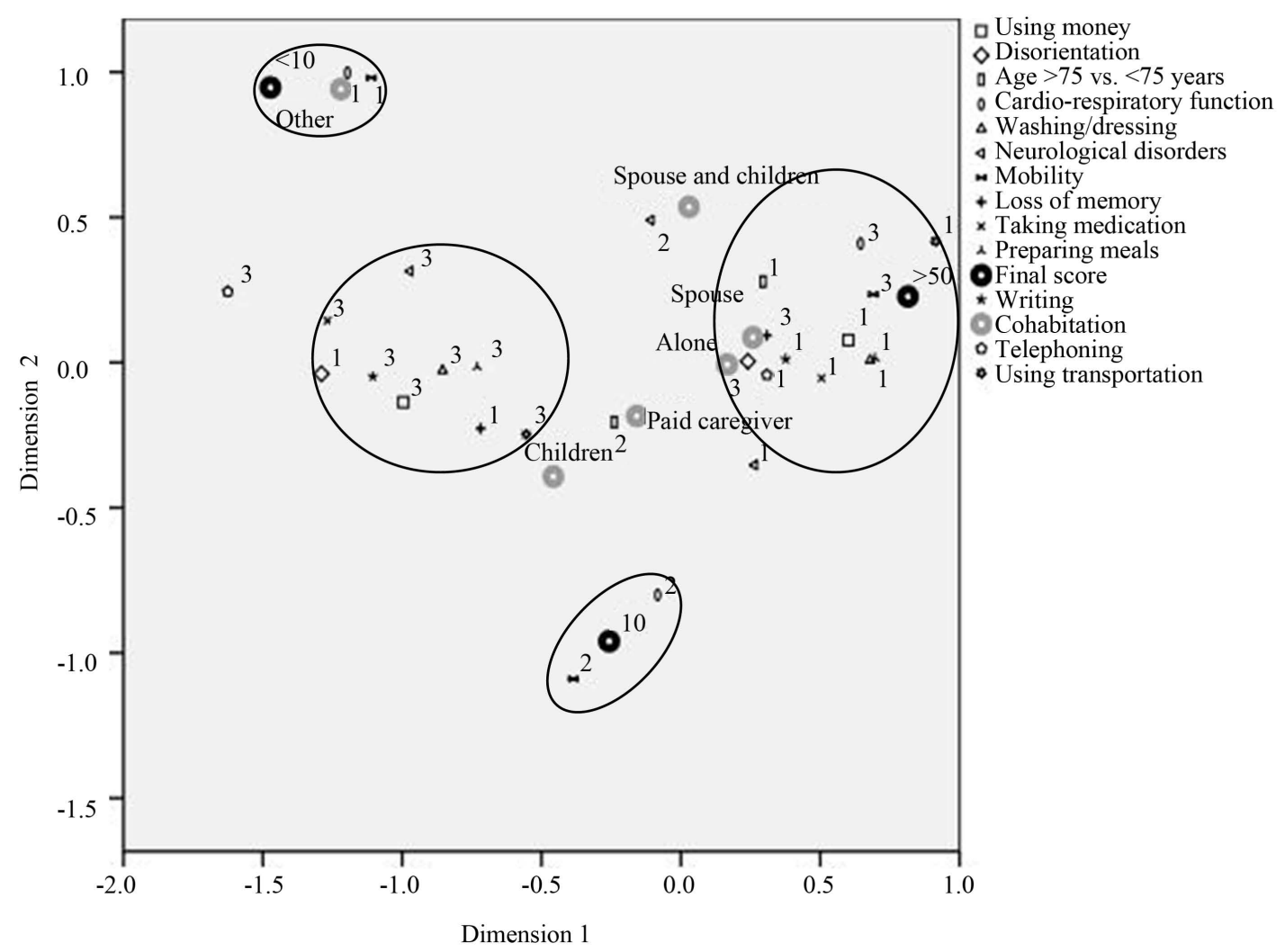

Graph 5. Multiple Correspondence Analysis (A score of 3 describes the worst condition and 1 the best condition for each variable. Instead the final score and type of cohabitation are separately stated). 


\section{Discussion}

Our study partially confirmed the factors reported by Bowles et al. [7] to identify the patients' requiring post-discharge care. In particular, they were important to determine their level of healthcare dependency: the presence of at least two neurological diseases and the lack of autonomy in carrying out IADLs (e.g., using public transport, preparing their meals, using money, taking medication, getting washed and dressed, and telephoning). Also the importance of social and financial resources emerged from our findings (e.g., availability of their children or of a paid caregiver), which in our study were associated with a serious lack of autonomy, and constituted an essential condition to avoid older patients from being institutionalised. At the same time, when older patients required post-discharge healthcare support and had no social and/or financial support, discharge was not a straightforward process and it needed to be planned very carefully. Specific tools were needed to have a comprehensive picture of all the aspects of their healthcare needs. Instead, a multidimensional assessment of older patients upon discharge permitted checking all the characteristics of the healthcare needs upon discharge and designing an appropriate post-discharge healthcare plan. This allowed optimizing the recovery process and reducing readmission.

Our results were very similar to those obtained with the Comprehensive Geriatric Assessment (CGA), a multidimensional and interdisciplinary diagnosing process aiming at determining the medical, psychological, and functional conditions of frail older patients, which could be used to develop long-term coordinated and integrated treatment and monitoring. Caplan et al. [13] highlighted the many advantages of CGA applied to older people discharged from the Emergency Department (ED). Gill et al. [14] underlined the importance of the financial implications and provided a simple cost-benefit analysis of the data produced by Caplan et al. [13]. In addition, the patients randomised to receive CGA presented a low hospital admission rates during the first month after their first post-discharge examination compared to those who did not receive it, with the additional advantage of avoiding the consequences of hospitalisation (such as, bed sores, delirium, and functional decline) in frail older patients.

Ellis et al. [15] confirmed that it was more likely that patients receiving CGA were still alive and living in their own homes after 6 months (OR 1.25, 95\% CI 1.11 to 1.42, P = 0.0002) or at the end of the healthcare plan (on average 12 months). In addition, they were less likely to be institutionalised (OR 0.79, 95\% CI 0.69 to 0.88 , $\mathrm{P}<0.0001)$, die or get worse.

Also Buurman et al. [16] presented the results of a randomised controlled trial (RCT) on the effectiveness of the "Transitional Care Bridge", a nurse-led multidimensional program consisting of a post-discharge intensive domiciliary 6-month follow-up of patients.

In fact, healthcare needs do not end upon discharge, but continue and a social-healthcare balance is needed to ensure the best possible quality of life. Instead, the healthcare process is sometimes abruptly interrupted upon discharge.

Popejoy et al. [17] examined the evolution of the healthcare policies regarding hospital discharge planning. The case management of individuals at risk was neither adopted nor reimbursed through public funds. More recently, Popejoy et al. [18] conducted a web-based descriptive study on discharge planning in the hospitals of Missouri. They identified issues regarding the difficulty of discharging patients requiring respiratory support, haemodialysis, chemotherapy, radiotherapy, negative-pressure wound therapy, and psychiatric support.

Also in Italy, despite that Multidimensional Assessment of older people is well-established; the Geriatric Assessment Units vary a great deal with regard to the composition and methodology used. Moreover, discharge planning in older patients is essential for the success of intermediate care, especially if we consider the financial burden of repeated short-term readmissions.

Undoubtedly, the first step in optimising healthcare resources has to be the provision of services that have a relatively low cost, such as a home care nursing plan, a domiciliary rehabilitation program, or remote monitoring, which allows preventing functional decline and repeated readmissions.

In other words, the financial assessment of healthcare interventions could help to identify priorities when healthcare resources are scarce, and moreover could influence the decision to fund innovative programs.

The methodology we proposed also allowed involving families in the decisions regarding their relatives, since home care was not proposed as an alternative but to support and integrate the care that family members provided and therefore an agreement needed to be reached. Managing the continuum of care between hospitals and the community did not merely have the purpose of reducing inappropriate hospital admissions.

Instead, the availability of an optimal discharge plan for older patients requiring alternative forms of care is 
crucial, especially if health policies push for shorter hospital stays, if life expectancy increases, and social and family resources diminish.

Nurses play an essential role in the management of this process of care continuum: in hospitals nurses have the tools to select those requiring "supported discharge" (continuity manager), in collaboration with community nurses who in turn collaborate with general practitioners and plan healthcare interventions, including those provided by other professionals, and also facilitate access to resources and aids that optimise the recovery of autonomy, and support and educate both patients and caregivers (case management).

\section{References}

[1] ISTAT (2011) Annuario Statistico Italiano 2011.

[2] Ministero della Sanità (1992) Progetto Obiettivo “Tutela della salute degli anziani 1991-1995” a stralcio del Piano Sanitario Nazionale 1992. Roma, 31-1-1992.

[3] Rubenstein, L.Z., Josephson, K.R., Wieland, G.D., English, P.A., Sayre, J.A. and Kane, R.L. (1984) Effectiveness of a Geriatric Evaluation Unit. A Randomized Clinical Trial. The New England Journal of Medicine, 311, 1664-1670. http://dx.doi.org/10.1056/NEJM198412273112604

[4] Deschodt, M., Wellens, N.I.H., Braes, T., De Vuyst, A., Boonen, S., Flamaing, J., Moons, P. and Milisen, K. (2011) Prediction of Functional Decline in Older Hospitalized Patients: A Comparative Multicenter Study of Three Screening Tools. Aging Clinical \& Experimental Research, 23, 421-426. http://dx.doi.org/10.1007/BF03325237

[5] Hoogerduijn, J.G., Schuurmans, M.J., Duijnstee, M.S., de Rooij, S.E. and Grypdonck, M.F. (2007) A Systematic Review of Predictors and Screening Instruments to Identify Older Hospitalized Patients at Risk for Functional Decline. Journal of Clinical Nursing, 16, 46-57. http://dx.doi.org/10.1111/j.1365-2702.2006.01579.x

[6] Ferioli, M., Ferraresi, A. and Volpato, S. (2008) Correlati clinici della dipendenza assistenziale nei pazienti anziani ospedalizzati. Giornale italiano di Scienze infermieristiche, 3, 9-14.

[7] Bowles, K.H., Holmes, J.H., Ratcliffe, S.J., Liberatore, M., Nydick, R. and Naylor, M.D. (2009) Factors Identified by Experts to Support Decision Making for Post Acute Referral. Nursing Research, 58, 115-122. http://dx.doi.org/10.1097/NNR.0b013e318199b52a

[8] Hoogerduijn, J., Buurman, B., de Rooij, S., Korevaar, J., Grobbee, D. and Schuurmans, M. (2010) ISAR-HP an Instrument Predicting Functional Decline in Older Hospitalized Patients. Journal of Clinical Nursing, 19, 1219-1225.

[9] Grauer, H. and Birnbom, F. (1975) A Geriatric Functional Rating Scale to Determinate the Need for Institutional Care. Journal of the American Geriatrics Society, 23, 472-476.

[10] Bartoli, A., Marazzi, M. C., Palombi, L., Mancinelli, S. and Magnano, M. (1990) La versione italiana della Scala della Funzionalità Geriatrica di H. Grauer. Prospettive Sociali e Sanitarie, 27, 4-9.

[11] Scarcella, P., Liotta, G., Marazzi, M.C., Carbini, R. and Palombi, L. (2005) Analysis of Survival in a Sample of Elderly Patients from Ragusa, Italy on the Basis of a Primary Care Level Multidimensional Evaluation. Archives of Gerontology and Geriatrics, 40, 147-156. http://dx.doi.org/10.1016/j.archger.2004.07.004

[12] Liotta, G., Scarcella, P., Mancinelli, S., Palombi, L., Cancelli, A. and Marazzi, M.C. (2006) The Evaluation of Care Needs in Elderly People: The Use of Geriatric Functional Evaluation Questionnaire. Annali di Igiene, Medicina Preventiva e di Comunita, 18, 225-235.

[13] Caplan, G., Williams, A., Daly, B. and Abraham, K.A. (2004) Randomized, Controlled Trial of Comprehensive Geriatric Assessment and Multidisciplinary Intervention after Discharge of Elderly from the Emergency Department- The DEED II STUDY. Journal of the American Geriatrics Society, 52, 1417-1423. http://dx.doi.org/10.1111/j.1532-5415.2004.52401.x

[14] Gill, S.S., Gibson, M. and Al-Hammadi, A. (2005) Economic Implication of Discharge of Elderly from the Emergency Department Trial Results. Journal of the American Geriatrics Society, 53, 1642. http://dx.doi.org/10.1111/j.1532-5415.2005.53487 11.x

[15] Ellis, G., Whitehead, M.A., O’Neill, D., Langhorne, P. and Robinson. D. (2011) Comprehensive Geriatric Assessment for Older Adults Admitted to Hospital. Online Publication Date: July 2011.

http://onlinelibrary.wiley.com/cochranelibrary/search

[16] Buurman, B.M., Parlevliet, J.L., van Deelen, B.A.J., de Haan, R.J. and de Rooij, S.E. (2010) A Randomised Clinical Trial on a Comprehensive Geriatric Assessment and Intensive Home Follow-Up after Hospital Discharge: The Transitional Care Bridge. BMC Health Services Research, 10, 296. http://dx.doi.org/10.1186/1472-6963-10-296

[17] Popejoy, L.L., Moylan, K. and Galambos, C. (2009) A Review of Discharge Planning Research of Older Adults 19902008. Western Journal of Nursing Research, 31, 923-947. http://dx.doi.org/10.1177/0193945909334855

[18] Popejoy, L.L., Galambos, C., Moylan, K. and Madsen, R. (2012) Challenges to Hospital Discharge Planning for Older Adults. Clinical Nursing Research, 21, 431-449. http://dx.doi.org/10.1177/1054773812436373 
Scientific Research Publishing (SCIRP) is one of the largest Open Access journal publishers. It is currently publishing more than 200 open access, online, peer-reviewed journals covering a wide range of academic disciplines. SCIRP serves the worldwide academic communities and contributes to the progress and application of science with its publication.

Other selected journals from SCIRP are listed as below. Submit your manuscript to us via either submit@scirp.org or Online Submission Portal.
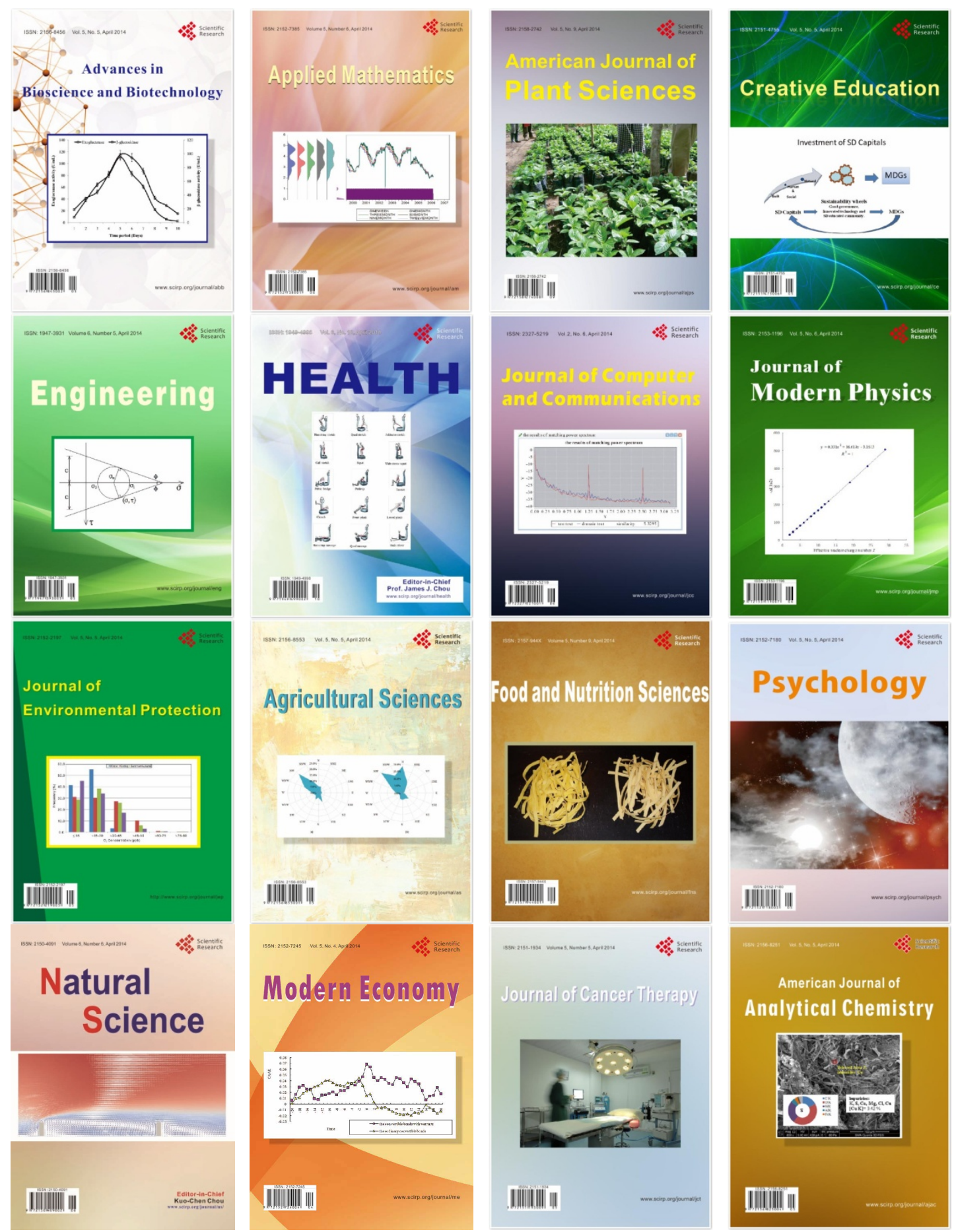